\title{
Pseudohypercreatininemia as an Initial Presentation of Waldenstrom's Macroglobulinemia
}

\author{
Tom T. Liu ${ }^{\mathrm{a}, \mathrm{b}}$, Mohebullah Zamkanei ${ }^{\mathrm{a}}$, Selina L. Liu ${ }^{\mathrm{c}}$, Jessica Liud, f, \\ Malvinder S. Parmar
}

\begin{abstract}
Chronic kidney disease (CKD) is a major public health problem. Estimated glomerular filtration rates based on serum creatinine values are used to diagnose this condition, and accuracy of laboratory testing is a crucial step in diagnosing and managing these patients efficiently in a timely manner. Spuriously elevated creatinine values have been reported due to the interference of other substances, including paraproteins. Paraproteins can interfere with the determination of various clinically important analytes in an unpredictable manner. While mild to moderate degrees of interference affecting the measurement of serum creatinine values have been previously reported, we present a case of a patient with markedly elevated serum creatinine who was referred for emergent assessment for possible consideration of renal replacement therapy. A thorough history and repeat laboratory investigations at several laboratories ultimately revealed the initial serum creatinine level to be spuriously high, i.e. "pseudohypercreatinimea" due to immunoglobulin interference. Further investigations ultimately led to the diagnosis of Waldenstrom's macroglobulinemia and the patient was appropriately treated. However, this case sheds light on the importance of being aware of a spuriously high creatinine value and its possible causes, as it can have significant diagnostic implications.
\end{abstract}

Keywords: Creatinine; Pseudohypercreatininemia; Assay interference; Waldenstrom's macroglobulinemia

\section{Introduction}

Chronic kidney disease (CKD) is a major public health prob-

Manuscript accepted for publication November 15, 2016

${ }^{a}$ Grand River Hospital, Kitchener, Ontario, Canada

${ }^{b}$ Faculty of Health Sciences, McMaster University, Hamilton, Ontario, Canada 'Division of Endocrinology \& Metabolism, Department of Medicine, Schulich School of Medicine \& Dentistry, Western University, London, Ontario, Canada dDepartment of Medicine, University of Toronto, 200 Elizabeth Street, 14EN213, Toronto, Ontario, Canada

${ }^{e}$ Clinical Sciences Division, Northern Ontario Medical School, Ontario, Canada

fCorresponding Author: Jessica Liu, Department of Medicine, University of Toronto, 200 Elizabeth Street, 14EN213, Toronto, Ontario, Canada.

Email: jessica.liu@uhn.ca

doi: https://doi.org/10.14740/wjnu282w lem, with a prevalence of about $12 \%$ in United States adults [1]. Accurate measurement of serum creatinine is important to diagnose and manage such high-risk patients effectively. Paraproteins can interfere in the determination of various clinically important analytes in an unpredictable manner affecting different methodologies [2-4]. Interferences of mild to moderate degree have been reported affecting the measurement of serum creatinine [5-8]. We present a patient with markedly elevated serum creatinine who was referred for consideration of emergent renal replacement therapy. Further investigations revealed this to be a case of falsely-elevated serum creatinine secondary to serum creatinine assay interference with an IgM monoclonal paraprotein, which led to the subsequent diagnosis of Waldenstrom's macroglobulinemia.

\section{Case Report}

A 70-year-old woman was emergently referred for consultation for advanced CKD with an elevated serum creatinine of $768 \mu \mathrm{mol} / \mathrm{L}$ (normal range (NR): $45-97 \mu \mathrm{mol} / \mathrm{L}$ ) and estimated glomerular filtration rate (eGFR, MDRD) of $5 \mathrm{~mL} /$ $\min / 1.73 \mathrm{~m}^{2}$. Her only symptom was mild fatigue. She denied symptoms of uremia, itching or shortness of breath. Her appetite was normal and weight was stable. Her exercise tolerance was reasonable. There was no history of hypertension or diabetes. She denied symptoms of joint pains or skin rashes. She denied any specific urinary symptoms and decrease in urine output or history of passage of discolored urine. She was on no medications and denied use of anti-inflammatory agents. Physical examination was unremarkable. Review of previous records revealed a normal serum creatinine of $69 \mu \mathrm{mol} / \mathrm{L} 5$ years prior.

To assess further, preliminary laboratory investigations were conducted at the hospital laboratory. The serum creatinine was normal at $73 \mu \mathrm{mol} / \mathrm{L}$. To rule out the possibility of a laboratory error because of the marked difference between the community and hospital laboratory values, concomitant outpatient and hospital samples were drawn and again, serum creatinine levels were within normal limits at the hospital laboratory. The urea was normal and there was no evidence of anemia, hyperkalemia or acidosis. A urinalysis was negative for protein and blood. A kidney ultrasound was normal. A technetium 99 m DTPA (diethylene-triamine-pentaacetate) renal scan showed a glomerular filtration rate (GFR) of $73 \mathrm{~mL} / \mathrm{min} / 1.73 \mathrm{~m}^{2}$. Taken together, the results from community laboratory were felt to be falsely elevated because of the assay interference. It was 

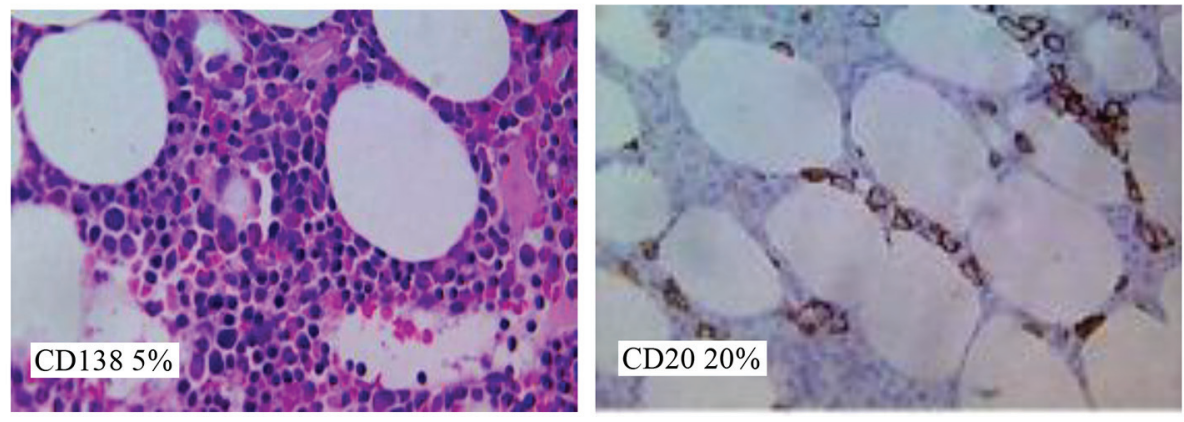

Figure 1. Bone marrow biopsy. Sections show a small core of hematopoietic marrow showing low-normal/decreased cellularity. There is evidence of trilineage hematopoiesis. Immunostatins show the presence of patchy mild CD20 positive and small B lymphoid infiltrate (approximately $20 \%$ overall), associated with mild increase in CD138 positive plasma cells (about $5 \%$, with formation of focal small aggregates). Overall picture is consistent with Waldenstrom's macroglobulinemia.

further confirmed by a normal serum creatinine concentration in the supernatant, after precipitation of the paraprotein with $3 \%$ sulfosalicylic acid.

The patient had a monoclonal IgM paraprotein with markedly elevated IgM level and the possibility of Waldenstrom's macroglobulinemia was considered, and as such, was referred to hematology. A bone marrow biopsy and aspirate confirmed the diagnosis and she responded well to chemotherapy (Fig. 1).

\section{Discussion}

Creatinine is a heterocyclic nitrogenous compound (IUPAC 2-amino-1-methyl-5H-imidazol-4-one, MW $113 \mathrm{Da}$ ). It is produced from creatine in muscle at a rate dependent on muscle bulk and is excreted unchanged by the kidneys, mainly by glomerular filtration but to a small extent by active secretion. Creatinine can be measured by different analytical methods - chemical, enzymatic or isotope-dilution mass spectrometry (ID-MS) with each having their benefits and limitations.

False elevations in serum creatinine levels (pseudohypercreatininemia), although rare, have been described [3, 6-10], including in patients with Waldenstrom's macroglobulinemia as initial presentation $[6,7]$, however, not to this degree of severity as in the present case requiring emergent workup. Salter et al [4] also reported similar intereference with serum creatinine measurement in two patients with IgM paraproteinemia where Siemens Advia enzymatic method was used.

As the enzymatic assays for the measurement of serum creatinine are commonly used and the paraproteinemias are not uncommon, vigilance and consideration of clinical context is required both by the clinicians and the laboratory staff when evaluating the cause of unexplained high serum creatinine. In this way, inappropriate diagnostic and therapeutic interventions may be prevented.

\section{Conflicts of Interest}

The authors have no conflicts of interest to disclose.

\section{References}

1. Coresh J, Byrd-Holt D, Astor BC, Briggs JP, Eggers PW, Lacher DA, Hostetter TH. Chronic kidney disease awareness, prevalence, and trends among U.S. adults, 1999 to 2000. J Am Soc Nephrol. 2005;16(1):180-188.

2. Roy V. Artifactual laboratory abnormalities in patients with paraproteinemia. South Med J. 2009;102(2):167170.

3. Kwok JS, Chow KM, Lit LC, Chan MH. Paraproteinemiaassociated pseudohypercreatininemia across different analytical methodologies. Kidney Int. 2010;78(6):621; author reply 621-622.

4. Salter T, Marsh J, Sood B, Livingstone C, Gallagher H. Pseudohypercreatininaemia in two patients caused by monoclonal IgM interference with enzymatic assay of creatinine. J Clin Pathol. 2015;68(10):854-855.

5. Datta P, Graham GA, Schoen I. Interference by IgG paraproteins in the Jaffe method for creatinine determination. Am J Clin Pathol. 1986;85(4):463-468.

6. Rudofsky G, Villalobos M, Waldherr R, Schaier M, Zorn M, Maimer A, Zeier M, et al. The Case: Renal failure in a male with Waldenstrom's macroglobulinemia. Kidney Int. 2010;77(4):371-372.

7. Storsley L, Fine A, Krahn J. IgM monoclonal protein presenting as pseudohypercreatininaemia. Nephrol Dial Transplant. 2006;21(11):3337-3338.

8. Ikeda M, Tsukamoto T, Miyake T, Kakita H, Komiya T, Yonemoto S, Muso E. [Case of pseudohypercreatininemia associated with monoclonal IgM gammopathy]. Nihon Jinzo Gakkai Shi. 2013;55(7):1340-1344.

9. Hummel KM, von Ahsen N, Kuhn RB, Kaboth U, Grunewald RW, Oellerich M, Muller GA. Pseudohypercreatininemia due to positive interference in enzymatic creatinine measurements caused by monoclonal IgM in patients with Waldenstrom's macroglobulinemia. Nephron. 2000;86(2):188-189.

10. Yamaki M. [A case of pseudohypercreatininemia in a patient suffering from macroglobulinemia]. Nihon Jinzo Gakkai Shi. 2004;46(1):43-48. 